\title{
The rise of quantitative methods in psychology
}

\author{
Denis Cousineau \\ Université de Montréal
}

\begin{abstract}
Quantitative methods have a long history in some scientific fields. Indeed, no one today would consider a qualitative data set in physics or a qualitative theory in chemistry. Quantitative methods are so central in these fields that they are often labelled "hard sciences". Here, we examine the question whether psychology is ready to enter the "hard science club" like biology did in the forties. The facts that a) over half of the statistical techniques used in psychology are less than 40 years old and that $b$ ) the number of simulations in empirical papers has followed an exponential growth since the eighties, both suggests that the answer is yes. The purpose of Tutorials in Quantitative Methods for Psychology is to provide a concise and easy access to the currents methods.
\end{abstract}

The use of agreed-upon quantitative methods is maybe the most reliable defining feature of the so-called "hard sciences". This trend was initiated by Descartes in the study of optics and, with a greater impact, by Galileo in the study of motion over four centuries ago. By 1905, the mutation they initiated fully matured, yielding among other, Einstein's relativity theory and Planck's quantum theory of the black box radiation. The quantum mechanic that evolved in the subsequent ten years was so mathematically involved (using the by-then weird matrix multiplication) that no vulgarization book appeared until the late 1940 (Hoffmann, 1947). Browsing the 1905 volume of the Physical Review, it is clear that qualitative measures and qualitative theories have completely disappeared from this field of research.

The same trend occurred in chemistry, following the works of Lavoisier, and Dalton in the eighteen century.

The most important lesson that we can get from this examination of the hard sciences evolution is that they were not born "hard sciences". Indeed, Socrates' theory of motion and the impetus theory were only qualitative. Hence, qualitative theory existed in physics for millennia (and maybe for as long as the human species!). Overall, physics took over 1500 years to mutate to a fully quantitative science.

Being the oldest science, physics offers a developmental scheme going from qualitative to quantitative. Kuhn would call these two stages, rather negatively, pre-scientific and scientific stages (Kuhn, 1996), although the so-called pre- scientific era of physics bolstered many scientific inquiries of high import.

A more neutral description of this mutation would emphasize the role of the tools used by the investigators. Physics evolved because it imported tools from mathematics and logic that allowed more powerful examination of theories. When those tools did not exist, they were created by researchers. During that time, these researchers were not specifically working on physic problems, but rather around those problems.

Paradoxically, it means that as a science evolves toward a hard science scheme, fewer scientists are actually probing the object of their discipline, as the development of tools and techniques requires more and more efforts.

Despite this division of labour, quantitative methods offer important gains:

a) Formalizing a method means that all the community agrees on the implementation details;

b) Increasing the precision of prediction allows for specific tests to be conducted with the possibility of falsifying a theory; and as a consequence,

c) Relying on empirical data, as opposed to the writings of influential predecessors.

Overall, the incorporation of quantitative methods lead to the accelerating rate of progress in hard sciences, which could be explained by the "law of accelerating returns" (Kurzweil, 1999). 


\section{Quantitative methods and psychology}

Does the developmental scheme that applied to physics apply as well to psychology? Not knowing what the future is made of, it is difficult to argue in one direction or the other. Further, the examination of physics is more like a case study. Hence, its scheme has no reason to apply to other fields such as psychology.

Some people might argue that quantitative methods are beneficial for psychology because it opens a greater number of research venues. In conjunction with the "law of increasing returns", it means that the more venues are explored, the more likely some of them will lead to fruitful developments. However, this line of reasoning is wrong. Indeed, prohibiting qualitative theories reduces the number of possible theories! Importing tools and techniques do increase the number of results though. Yet, it is not clear that more results means a progress for a scientific field (although they seem correlated).

Because argumentation is difficult, if not impossible, we propose instead to look at the current practice, how psychologists are investigating psychology.

The most important class of techniques used in or developed for psychologists are the statistical techniques. Of the hundred of statistical techniques, only a handful definitely have more than a hundred years (the z-test, based on Gauss, 1812, theory of errors, and the binomial test, maybe the oldest, dating back to the seventieth century). The correlation coefficient and the $\chi^{2}$ test were laid down near 1900 by Pearson. The 1900-1920 period saw the t-test (Student, 1908), the ANOVA (Fischer, 1925) and a nonparametric correlation coefficient (Spearman, 1904). In comparison, the 40 s and 50s saw a dozen of new tests (posthoc tests, repeated-measure ANOVA, etc.). However, a considerably larger number of tests were developed past 1965 (e. g. all the multivariate tests). It is difficult to have an exact quantification, but we estimate that roughly $50 \%$ of the statistical tests currently existing were developed in the last 40 years (although the most commonly used tests, ANOVA, t-test, regressions, are older). And this trend is not slowing down, with very popular techniques (such as Hierarchical Linear Model, Arnold, 1992, Structural Equation Modeling, Byrne, 1996 and Independent Component Analysis, Hyvarinën, Karhunen and Oja, 2001) all having less than 20 years of existence.

Another class of techniques used in psychology is the modelization of results. For instance, Golden (2000), searching the PscyhInfo database, found an exponential growth in the use of neural networks: Whereas a dozen of papers were modeling psychological results with neural networks prior to 1985 , this number has gone to half a thousand in the subsequent decade, and reaches tens of thousands between 1995 and 2005 (our search).

Similar, although less impressive, results are found with the sampling models (including the signal detection theory), a class of models generally distinct from the neural networks models (but see Cousineau, 2004): Their use in psychology started in the 60s; they really exploded in the 80s.

Likewise, we can consider instruments. There is a considerably increase in the number and precision of datagathering devices. In the 50s, visual stimuli requiring specific duration times were either presented with a tachitoscope or a cathode-ray tube. For the latter, simply presenting one of a couple of letters required months to realize the suitable circuit! Because it was relatively easier to play tapes, auditory experiments were favoured in the 50s. Nowadays, an experiment that needed over six months to assemble with tapes and wires in the 60s can be designed in less than an hour with the current tools (often software tools).

Taken as a whole, the picture shows a strong surge of quantitative modeling in psychology. There are still psychologists generating qualitative theories but they generally use statistical tools on their data. Hence, the question raised at the beginning of this section (will psychology become a hard science) can empirically be answered: psychology is becoming right now, under our own eyes, a hard science. Hence, this new question: As psychology researchers, are we ready for this mutation?

\section{Tutorials in Quantitative methods}

By analogy with the astrology that gave rise to astronomy, some psychologists suggested that fundamental psychology (as opposed to pop psychology, Larivé, 2004) adopt a new name, psychonomy. Although I don't see the need for a change of name, it is clear that the practice of the psychologists is changing. We estimate that during graduate studies, a student spends $50 \%$ of the time mastering techniques that are necessary but peripheral to the theme of the research. This is a lot of time devoted to "how-to" learning. And this ratio may only go worse.

One approach is to change the undergraduate course contents to cover more of these tools (Giguère, Hélie, \& Cousineau, 2004). Another approach is to increase the ease with which researchers can access and master those techniques.

This is the purpose of the Tutorials in Quantitative Models for Psychology (TQMP). We believe it is important to provide quick and concise access to recent technologies so that we can spend more time on the essence of our research. The less time we devote learning a technique, the faster we can start using it, the better it is for psychology as a scientific 
discipline.

Papers submitted to TQMP must first be a clear and comprehensive tutorial for a specific technique. Because there is no better way to learn than to try, it should also come with a data set (likely fictitious data) downloadable from the journal's web site. The manuscript should also indicate potential pitfalls or difficulties and indicate in what situations the method should or should not be used. Finally, if it requires programming, a minimal program should also be provided and briefly explained in the manuscript. In this respect, high-level programming languages (such as Mathematica, Matlab, R) should be preferred over low-level programming languages (such as $\mathrm{C}$, Basic, Fortran). In addition, platform-independent programming should be favoured as well. Finally, the tutorials should be short but explicitly list references for more thorough expositions, whenever they exist. The reference list can be commented to indicate the level of difficult of the suggested reading.

\section{References}

Arnold, C. L. (1992). Methods, plainly speaking: An introduction to hierarchical linear models. Measurement and evaluation in counseling and development, 25, 5890.

\section{Introduction to HLM.}

Byrne, B. M. (1994). Structural equation modeling with eqs and eqs/windows: Basic concepts, applications and programming. Thousand oaks: Sage.

Very accessible introduction to EQS.

Cousineau, D. (2004). Merging race models and adaptive networks: A parallel race network. Psychonomic Bulletin \& Review, 11, 807-825.

Fisher, R. A. (1925). Applications of the Student's distribution. Metron, 5, 3-17.
Gauss, C.F. (1809/1864). Theoria Motus Corporum Coelestium in Sectionibus Conicis Solem Ambientum. Paris: A. Bertrand.

Giguère, G., Hélie, S., \& Cousineau, D. (2004). Manifeste pour le retour des sciences En psychologie. Revue Québécoise de Psychologie, 25, 117-130.

Golden, R. M. (2001, July). Statistical hypothesis testing in complex behavioral simulation models. Society for Mathematical Psychology, Providence, RI.

Heathcote, A. \& Mewhort, D. J. K. (1993). Representation and selection of relative position. Journal of Experimental Psychology: Human Perception and Performance, 19, 488-516.

Hoffmann, B. (1947). The strange theory of the quantum. New York: Dover publications.

Funny to read as the author spend so much time explaining that in linear algebra, A.B $\neq$ B.A.

Hyvärinen, A., Karhunen, J. \& Oja, E. (2001). Independent Component Analysis. New York: John Wiley and sons.Kuhn, T. S. (1996). The Structure of Scientific Revolutions (3rd edition). Chicago: The University of Chicago Press.

\section{A technical introduction to ICA.}

Kurzweil, R. (1999). The age of spiritual machines. New York: Penguin books.

Very inspiring book for all audience although propably over-optimistic.

Larivée, S. (2004). Pour éviter de nuire, une nouvelle rubrique. Revue de psychoéducation, 33, 1-14.

Spearman, C. E. (1904). Proof and measurement of association between two things. American Journal of Psychology, 15, 72-101.

Student (1908). The probable error of a mean. Biometrika, 6, 1-25.

A classic easily found on the web.

Received August 6, 2005 